\title{
Oroxin B Attenuates Ovariectomy-Induced Bone Loss by Suppressing Osteoclast Formation and Activity
}

\author{
Jun-ming Huang (D) \\ Chen-zhong Wang \\ Shun-yi Lu \\ Zhe Wang \\ Zuo-qin Yan
}

Department of Orthopaedics, Zhongshan Hospital, Fudan University, Shanghai, 200032, People's Republic of China
Correspondence: Zuo-qin Yan 180 Feng Lin Road, Xuhui District, Shanghai, 200032, People's Republic of China

Email yan.zuoqin@zs-hospital.sh.cn
Background: Osteoclasts are the major players in bone resorption and have always been studied in the prevention and treatment of osteoporosis. Previous studies have confirmed that a variety of flavonoids inhibit osteoporosis and improve bone health mainly through inhibiting osteoclastogenesis. Oroxin B (OB) is a flavonoid compound extracted from traditional Chinese herbal medicine Oroxylum indicum (L.) Vent, exerts potent antitumor and antiinflammation effect, but its effect on osteoclastogensis remains unknown.

Methods: We comprehensively evaluated the effect of $\mathrm{OB}$ on the formation and function of osteoclasts and the underling mechanism by bone marrow-derived macrophage in vitro. In vivo, we used mice ovariectomized model to verify the protective effect of OB.

Results: OB was found to inhibit osteoclast formation and bone resorption function in vitro, in a dose-dependent manner and the increased osteoclastic-related genes induced by RANKL (NFATc1, c-fos, cathepsin K, RANK, MMP9 and TRAP) were also attenuated following OB treatment. Mechanistical investigation showed $\mathrm{OB}$ abrogated the increased phosphorylation level of MAPK and NF- $\mathrm{B}$ pathway, and diminished the expression of the vital transcription factors for osteoclastogenesis. OB also prevented ovariectomy (OVX)-induced bone loss by inhibiting osteoclast formation and activity in mice.

Conclusion: Our study demonstrated that OB may act as an anti-osteoporosis agent by inhibiting osteoclast maturation and attenuating bone resorption.

Keywords: osteoclast, Oroxin B, MAPK, NF-кB, RANKL, osteoporosis

\section{Introduction}

Osteoporosis is a common systemic metabolic bone disease featured with reduced bone mass, deformed microarchitecture, and decreased bone strength, leading to increased bone fragility and susceptibility to fracture. According to reports, 200 million people worldwide suffer from osteoporosis and 9 million have fractures due to osteoporosis. ${ }^{1,2}$ Although there are significant differences in the incidence of osteoporosis among different regions, genders and age groups, the incidence of osteoporosis is greatly increased in people over 60 years, especially in women. With the accelerated pace of the global aging society, the total number of aging populations will further rise, followed by a substantial increase in the number of patients with osteoporosis. Therefore, the prevention and treatment of osteoporosis is an urgent concern. During the past three decades, a variety of medications have been used for the prevention and treatment of osteoporosis, but few are entirely satisfactory. Bisphosphonates are popular and effective in reducing bone mass loss, 
but long-term usage may lead to atypical femoral fractures and necrosis of jaw bone and estrogens. Hormone replacement therapy (HRP), is only recommended for women who also have menopausal symptoms and selective estrogen receptor modulator raloxifene may increase the risk of blood clot and strokes.

The maintenance of bone homeostasis is fine-tuned by two highly coupling aspects, namely bone formation and bone resorption. ${ }^{3}$ The uncoupling of the bone resorption/ formation process is critical to the development of osteoporosis, with an exaggeration of resorption, reduction in bone formation or a combination of both. In postmenopausal osteoporosis, abnormal increased resorption or demineralization are the main causes of osteoporosis. Osteoclasts are large multinucleated cell, which are differentiated from the monocyte-macrophage lineage precursor cells, and are the main type of cell responsible for resorbing bones. In the bone microenvironment, several cytokines were reported to contribute osteoclast differentiation, among which the two cytokines, macrophage colonystimulating factor (M-CSF) and receptor activator of nuclear factor-kappa B (NF-kB) ligand (RANKL), are demonstrated as the most crucial factor. During osteoclast differentiation, M-CSF not only promotes proliferation and survival of bone marrow monocytes (BMMs) and osteoclast precursors but also stimulates the expression of RANK, increasing activity of the RANK and RANKL complex by binding to CSF1R. ${ }^{4,5}$ RANKL is a transmembrane molecule and belongs to the tumor necrosis factor cytokine family. During osteoclast differentiation, RANKL binds to RANK and stimulates NF- $\mathrm{KB}$ and mitogen-activated protein kinases (MAPKs) signaling pathways and then the activated signaling pathways upregulate and activate downstream transcription factors, c-fos and activated $\mathrm{T}$ cell cytoplasm 1 (NFATc1), which are the hub molecules controlling the differentiation of osteoclasts. $^{6,7}$ Following the activation of $\mathrm{c}$-fos and NFATc1, the main molecules responsible for bone resorption (tartrate-resistant acid phosphatase (TRAP), cathepsin $\mathrm{K}$ and matrix metalloproteinase 9 (MMP9)) are upregulated and ultimately completes bone resorption.

In China, the cultivation and use of medicinal plants play a very important role in traditional systems of medicines, and these plants are considered the primary sources of pharmaceutical compounds. ${ }^{8}$ Studies involving natural herbal products have been increasingly popular to inhibit osteoclast formation and treat osteoclast-related diseases. Flavonoids exist naturally in fruits, vegetables, grains, tree bark, roots, stems, flowers, tea, and wine. ${ }^{9}$ These natural products were widely known for their health benefits long before flavonoids with variable phenolic structure were isolated. Presently, several thousand flavonoids have been extracted and identified with most of them possessing a wide range of beneficial effects on antioxidation, cancer, diabetes, vascular and nervous diseases. ${ }^{10}$ In skeletal system, the regulatory effect of flavonoids on bone metabolism has been widely reported. Icarrin, the most abundant flavonoid constituent in traditional Chinese herb Epimedii, has been shown to stimulate bone regeneration and repair and demonstrated as alternative treatment for osteoporosis in clinical practices. ${ }^{11-13}$ In addition, some other flavonoids, proanthocyanidins, eriodicyol, baicalin and so on, were reported to attenuate OVX-induced bone loss. ${ }^{14-16}$

Oroxin B (OB), monomer composition, is one of the flavonoids extracted from traditional Chinese herb Oroxylum indicum (L.) Vent. However, at present, only four articles reported the biological function of OB. Firstly, OB was confirmed to markedly inhibit the hemolytic activity of $\alpha$-Hemolysin and then Yang et al demonstrated that OB had anti-lymphoma effect without toxicity through inducing tumor-suppressive ER stress and inhibiting tumor-adaptive ER stress. ${ }^{17,18}$ The two recently published studies have confirmed that $\mathrm{OB}$ effectively exerts anti-liver cancer activity by inducing apoptosis in cancer cells. ${ }^{19,20}$ However, there is no study evaluating the effect of OB on the skeletal system. In this study, we aim to evaluate the effect of $\mathrm{OB}$ on osteoclast generation and activation and to explore the potential underlying mechanism in vitro, and its potential therapeutic ability in an osteoporosis model with estrogen withdrawal.

\section{Materials and Methods}

\section{Reagents}

Oroxin B (OB; $\mathrm{C}_{27} \mathrm{H}_{30} \mathrm{O}_{15} ;$ MW: 594.52) and Cell Counting Kit-8 (CCK-8) were purchased from Target Molecule Corp. (Boston, MA, USA), recombinant mouse RANKL and M-CSF from PeproTech (Princeton, NJ, USA), DAPI and Actin-Tracker Green from Beyotime (Shanghai, China). Other reagents were of the highest commercial grade and were purchased from SigmaAldrich (St. Louis, MO, USA). Oroxin B was diluted in DMSO to different concentrations $(10 \mathrm{mM}, 20 \mathrm{mM}, 30 \mathrm{mM}$, $40 \mathrm{mM}, 50 \mathrm{mM}$ ). In vitro, the diluted $\mathrm{OB}$ is added to the medium at a ratio of 1:1000 and an equal volume of DMSO was added to the control group. 


\section{Cell Culture}

Bone marrow-derived macrophages (BMM) were isolated from the tibia and femurs of 6 -week-old male C57BL/6 mice as previously described. ${ }^{21}$ Femurs and tibias were removed from mice and stripped off the soft tissue. Bone marrow suspensions were isolated from bone marrow cavities of 6-week=old mice tibias and femurs by flushing with $\alpha$-MEM medium containing $15 \%$ fetal bovine serum (FBS). On the following day, the cells were collected from the suspension and cultured in medium supplemented with 30 $\mathrm{ng} / \mathrm{mL} \mathrm{M}-\mathrm{CSF}$ to remove bone marrow stromal cells, and nonadherent cells (BMMCs) were collected for subsequent use. In all experiments, M-CSF (30ng/mL) was used to maintain the survival of BMMs. RANKL $(50 \mathrm{ng} / \mathrm{mL})$ was used to stimulate osteoclast differentiation.

\section{Cell Counting}

The CCK-8 assay was used to evaluate the cytotoxic effects of OB on BMMs. BMMs were seeded at density of $5 \times 10^{3}$ cells/well in 96-well plates. Twenty-four hours later, the BMMs were treated with DMSO (vehicle) and different concentrations of $\mathrm{OB}(10 \mu \mathrm{M}, 20 \mu \mathrm{M}, 30 \mu \mathrm{M}$, $40 \mu \mathrm{M}, 50 \mu \mathrm{M})$. After $1,3,5$ and 7 days, fresh medium containing 1/10 cell CCK-8 reagent was added and then incubated at $37^{\circ} \mathrm{C}$ for 1 hour. The absorbance of each well was recorded by FlexStation 3 at a wavelength of $450-\mathrm{nm}$ (Molecular Device, Shanghai).

\section{LIVE/DEAD Viability Assay}

According to instruction manual of LIVE/DEAD Cell Imaging Kit (R37601) (Thermo Fisher Scientific, Waltham, MA, USA), the live green vial (A) was transferred into dead red vial (B) to create a $2 \mathrm{X}$ stock. Equal volume of the $2 \mathrm{X}$ stock was added to the culture plate ensuring full coverage of cells and observed under fluorescence microscope after incubation at $25^{\circ} \mathrm{C}$ for $15 \mathrm{~min}$ away from light. Live and dead cells are represented by the colors green and red.

\section{In vitro Osteoclast Differentiation Assay}

The BMMs were seeded at density of $1 \times 10^{4}$ cells/well in 96-well plates. After 24 hours, RANKL was added into the medium in combination with different concentrations of OB $(10 \mu \mathrm{M}, 20 \mu \mathrm{M}, 30 \mu \mathrm{M}, 40 \mu \mathrm{M}, 50 \mu \mathrm{M})$, while $50 \mu \mathrm{M}$ OB was supplemented in medium on either day 1 or day 3 . After 5 days, the formed osteoclasts were stained by tartrate-resistant acid phosphatase (TRAP) staining kit. TRAP-positive multinucleated cells with three or more nuclei were counted as osteoclast. Imaging was then acquired using Olympus IX-71 (OLYPUMS, Japan).

\section{Actin Staining}

The BMMs were seeded at a density of $2 \times 10^{4}$ cells/well in 96-wells plate cultured with M-CSF. Twenty-four hours later, RANKL and $\mathrm{OB}$ were added into the medium. After 5 days, mature osteoclasts were observed and fixed with 4\% PFA for 20 minutes. Osteoclasts were then stained with Actin-Tracker Green for 1 hour at room temperature and DAPI was used to identify cellular nuclei. Imaging was then done using a fluorescent microscope.

\section{Pit Formation Assays}

The BMMs were seeded at a density of $2 \times 10^{4}$ cells/well in Corning Osteo Assay Surface plate (Corning Incorporated Life Science, NY, United States). The BMMs were cultured with M-CSF and RANKL for 5 days. After mature osteoclast formation, different concentrations of OB were added to the medium containing M-CSF and RANKL for 3 days. The disks were treated with $5 \%$ sodium hypochlorite for 5 minutes, and images were taken using a light microscope. The resorption pits were quantified using ImageJ.

\section{RNA Extraction and Quantitative Real-Time PCR}

BMMs were seeded at a density of $2.5 \times 10^{5}$ cells/well in 6-well plates and cultured with M-CSF. Twenty-four hours later, RANKL and OB were added into medium. Three days after stimulation, the total RNA was extracted by using RNA-Quick Purification Kit (YISHAN Bio-Tec, Shanghai). The total RNA $(1 \mu \mathrm{g})$ was mixed with the PrimeScript Reverse Transcriptase Master Mix kit (TaKaRa, Japan) to synthesize cDNA. Then, the synthesized cDNA was then used to perform RT-qPCR, by using SYBR qPCR Mix (Ysasen, Shanghai) on Thermo Fisher Scientific Q5 instrument. The relative expression of target genes was calculated and normalized to the reference gene $\beta$-actin. The primers used in RT-qPCR are presented in Table 1.

\section{Western Blot Analyses}

To explore the mechanism of $\mathrm{OB}$ on osteoclast differentiation, BMMs were seeded on 6-well plates $\left(2.5 \times 10^{5}\right.$ cells/well $)$ for 24 hours. Then, the cells were pretreated with $\mathrm{OB}(50 \mu \mathrm{M})$ in FBS-free medium for 12 hours and then stimulated with RANKL for $5 \mathrm{~min}, 15 \mathrm{~min}$ and $45 \mathrm{~min}$, respectively. To analyze the long-time action of $\mathrm{OB}$ on osteoclastogenesis, 
Table I List of Primers Used in Quantitative Real-Time RT-PCR

\begin{tabular}{|l|c|c|}
\hline Target Gene & Sense Sequence (5'-3') & Antisense Sequence (5'-3') \\
\hline NFATcl & CAACGCCCTGACCACCGATAG & GGGAAGTCAGAAGTGGGTGGA \\
Rank & CAGGAGAGGCATTATGAGCA & GGTACTTTCCTGGTTCGCAT \\
TRAP & TACCTGTGTGGACATGACC & CAGATCCATAGTGAAACCGC \\
Cathepsin $K$ & TGTATAACGCCACGGCAAA & GGTTCACATTATCACGGTCACA \\
c-Fos & CGGGTTTCAACGCCGACTA & TTGGCACTAGAGACGGACAGA \\
MMP9 & CTGGACAGCCAGACACTAAAG & CTCGCGGCAAGTCTTCAGAG \\
$\beta$-Actin & GGCTGTATTCCCCTCCATCG & CCAGTTGGTAACAATGCCATGT \\
\hline
\end{tabular}

BMMs were seeded at density of $2.5 \times 10^{5}$ cells/well in 6-well plates and cultured with M-CSF. Twenty-four hours later, RANKL and OB were added to the medium. After 3 days stimulation, the RIPA lysis solution containing broad spectrum phosphatase inhibitors ( $1 \mathrm{mM})$ and PMSF (1mM) was used to prepare cell lysates. The concentration of each sample was measured by BCA assay kit (Thermo Fisher Scientific, MA, USA). In Western blot, equal proteins $(10 \mu \mathrm{g})$ were subjected to $10 \%$ SDS polyacrylamide gel and transferred to the PVDF membranes (Millipore, MA, United States). The Protein Free Rapid Blocking Buffer (EpiZyme, Shanghai) was used to block the membranes followed by incubated with respective antibodies overnight at $4^{\circ} \mathrm{C}$. The membranes were washed with TBS-Tween and then incubated with HRP-conjugated secondary antibodies at room temperature. Subsequently, the membranes were immersed in western ECL Substrate Kits (Yseasen, Shanghai) and the protein bands were detected using Tanon imaging system. Analysis of the grayscale image were obtained using ImageJ software.

Antibodies were purchased from Cell Signaling Technology (Boston, MA, USA): p38 (\#8690), p-p38 (\#4511), ERK (\#9102), p-ERK (\#4370), JNK (\#9252), p-JNK (\#4668), p65 (\#8242), p-p65 (\#3033), IкB $\alpha$ (\#4812), $\mathrm{p}-\mathrm{I} \kappa \mathrm{B} \alpha$ (\#2859), c-fos (\#2250) and RANK (\#4845). The antibody to NFATc1 (66963-1-Ig), and $\beta$-actin (66009-1-Ig) were purchased from Proteintech Group Inc. (Wuhan, China). The antibody to cathepsin K (DF6614) and MMP9 (AF5228) were purchased from Affinity Biosciences (USA). Anti-rabbit and anti-mouse HRP-conjugated secondary antibodies were obtained from Jackson ImmunoResearch Inc. (West Grove, PA, USA).

\section{Immunofluorescence Staining}

BMMs were seeded at a density of $1 \times 10^{4}$ cells/well in 96well plates and cultured with M-CSF. Twenty-four hours later, RANKL and $\mathrm{OB}$ were added into medium. After 3 days, cells were fixed with 4\% PFA (20min) and permeabilized with $0.25 \%$ Triton X-100 (5min). Osteoclasts were blocked using $2 \%$ BSA for 1 hour at room temperature and then incubated using the antibody against p65 or NFATc1 overnight at $4^{\circ} \mathrm{C}$. On the next day, secondary antibody conjugated with FITC or Cy3 (Boster, Wuhan) was selected for incubating at room temperature for 1 hour. Actin-Tracker Green was used for marking actin (1hour) and DAPI was used to mark cell nuclei (5min). The fluorescent images were captured by using fluorescence microscope or laser scanning confocal microscope.

\section{Animals}

Animal care and experimental procedures were approved by the Animal Use and Care Committee of Zhongshan hospital, Fudan University (Shanghai, China) and following the guidelines of the NIH "Principles of Laboratory Animal Care" (1996 Revised Version). Twelve-week-old female C57/BL6 mice (28.7 \pm 0.8$)$ were provided by SLAC Laboratory Animal Co. Ltd (Shanghai, China), and maintained in the animal care facility of Fudan University at $25^{\circ} \mathrm{C}$ with $12: 12$ light/dark cycle. They were randomly distributed into three groups ( $\mathrm{n}=8 \mathrm{mice} /$ group): shamoperated mice intraperitoneal injected with equal volume DMSO (SHAM), bilateral ovariectomized mice intraperitoneal injected with DMSO (OVX), bilateral ovariectomized mice intraperitoneal injected with $\mathrm{OB}(40 \mathrm{mg} / \mathrm{kg})$ (OB) similar to previous studies. ${ }^{18} \mathrm{OB}$ and DMSO were administered every 2 days for 6 weeks starting on the third day after surgery. After 6 weeks, all the mice were sacrificed by an intraperitoneal injection of $10 \%$ chloral hydrate and harvested for study.

\section{Micro-Computed Tomography ( $\mu$-CT) and Histomorphometric Analysis}

After fixation and removal soft tissue, all femoral specimens were scanned using Scanco vivaCT 40 instrument (Scanco Medical, Basserdorf, Switzerland). Serial 10.5- $\mu \mathrm{m}$ 
tomographic images were acquired at $100 \mathrm{kV}$ and $98 \mathrm{~mA}$. Then, the 3D images were obtained and observed from sagittal, coronal, and transverse position by built-in software in the $\mu$-CT system. Four morphometry parameters: bone volume/ tissue volume (BV/TV), trabecular number (Tb.N), trabecular thickness (Tb.Th) and trabecular separation (Tb.Sp), were quantitatively measured using the built-in software. Following micro-CT analysis, all femurs were decalcified at room temperature for 3 weeks. All samples were then were embedded in paraffin and sectioned on a microtome at a thickness of $5 \mu \mathrm{m}$. Hematoxylin and eosin (H\&E) staining was used to evaluate the the trabecular structure. TRAP staining was performed and the number of osteoclasts near femoral metaphysis were counted according to a previous study. ${ }^{22}$ Bone sections were captured at $200 \times$ magnification. TRAPpositive cells that formed resorption lacunae at the surface of the trabeculae and contained one or more nuclei were identified as osteoclasts.

\section{Serum Biochemistry}

Blood was collected from mice and serum was separated by centrifuging immediately. Serum levels of type 1 collagen cross-linked C-terminal telopeptide (CTX-I), osteoprotegerin (OPG) and RANKL was evaluated by ELISA kit (Boster, Wuhan, China).

\section{Statistical Analysis}

All experiments were repeated independently for 3 times and the data were expressed as mean \pm SD. The Q-Q plot demonstrated that the animal data distribution was skewed, thus data were described with median (min-max) (Supplemental Material). Statistical analysis was conducted by nonparametric test such as Mann-Whitney test and KruskalWallis test. The difference between the two groups was verified by Mann-Whitney test. The Kruskal-Wallis rank sum test was applied to test the data obtained from animals. Pairwise comparisons were conducted after Kruskal-Wallis test as post-hoc tests. Statistical analyses were carried out using the calculation software in GraphPad Prism and SPSS 23.0. P values less than 0.05 were considered statistically significant.

\section{Results \\ OB Attenuates RANKL-Induced Osteoclast Differentiation in vitro}

We measured the potential cytotoxicity of OB on BMMs by CKK-8 assay at day 1 , day 3 , day 5 , and day 7 . OB concentration up to $50 \mu \mathrm{M}$ showed no negative effect on BMMs proliferation (Figure 1A). In addition, live/dead viability assay showed that $\mathrm{OB}$ had no significant effect on cell viability after 7-day treatment (Figure 1B). We further tested the effect of OB on RANKL-induced osteoclast formation and we found OB inhibited the osteoclast' formation in a dose-dependent manner (Figure 2A and $\mathrm{C}$ ). To investigate the time course effect of $\mathrm{OB}$ on osteoclast formation, $50 \mu \mathrm{M}$ OB was added in culture for a specified number of days during RANKLstimulated osteoclast differentiation. When OB was added in the early stage of osteoclast differentiation (day 1 to day 3 ), the osteoclast formation was significantly reduced (Figure $2 \mathrm{~B}$ and D). However, when BMMs were exposed to $\mathrm{OB}$ on day 3 to day 5 , the number of TRAP-positive osteoclasts showed a slight reduced, indicating that $\mathrm{OB}$ impaired early stages of the osteoclast formation process.

\section{OB Exhibits Anti-Resorption Effect in vitro}

During osteoclast differentiation, F-actin rings are considered as the characteristic structure of mature osteoclasts and is indispensable for its bone resorption activity. ${ }^{23}$ In this study, we used Actin-Tracker to identify the formation and morphology of F-actin. Consistent with inhibition of osteoclast formation, the formation of podosomal actin belts was disrupted and the reduced actin ring formation was associated with intervention concentration of $\mathrm{OB}$ (Figure 3A). Functionally, osteoclasts are the only type of cell responsible for bone resorption. Thus, we tested the osteoclast bone resorptive function after administration of $\mathrm{OB}$. The reabsorbed area of the bone plate was reduced when osteoclasts were treated with $\mathrm{OB}$ (Figure 3B and C).

\section{OB Abrogates RANKL-Associated NFATcl Activation, and Downregulates Osteoclast-Related Genes}

During RANKL-induced osteoclastogenesis, c-fos and NFATc1 are key transcription factors for osteoclast differentiation and the expression of cathepsin K, MMP9 and TRAP are the prerequisites of bone resorption. Consistent with osteoclast formation and bone resorption, OB treatment decreased the gene expression of Rank, c-fos, NFATc1, cathepsin K, MMP9 and TRAP in a dosedependent manner (Figure 4A). Corresponding to the mRNA results, the protein expression of c-fos, cathepsin K, MMP9 and NFATc1 was significantly hampered by administration of $\mathrm{OB}$ by immunoblot analysis (Figure 4B-E). Immunofluorescence staining OB treatment blocks NFATc1 function (Figure 4F). 


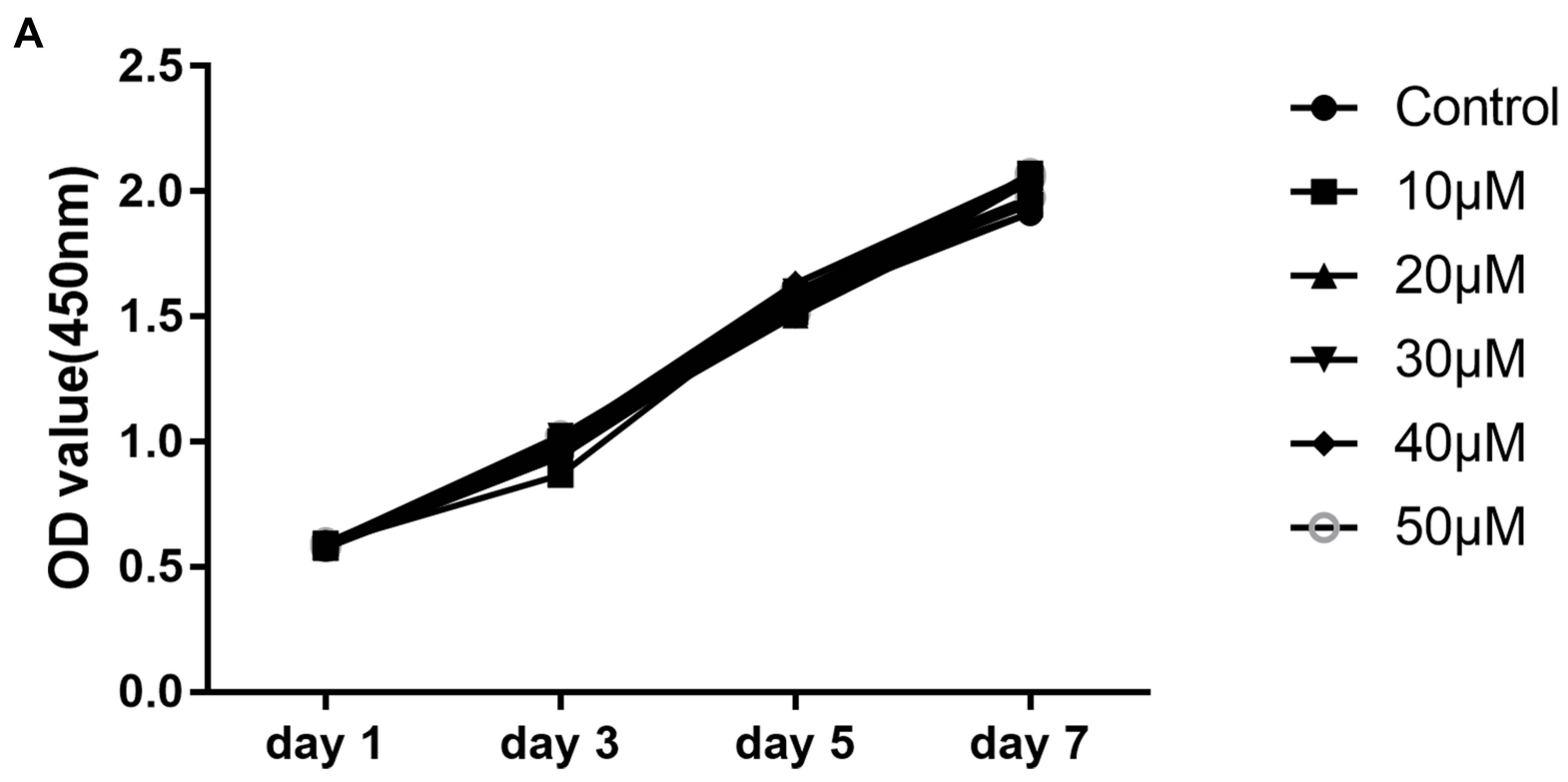

B

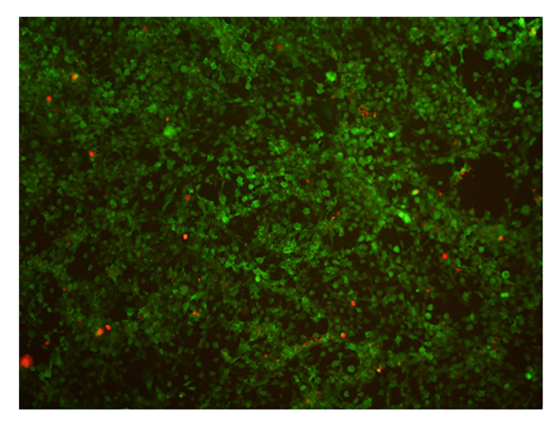

Control

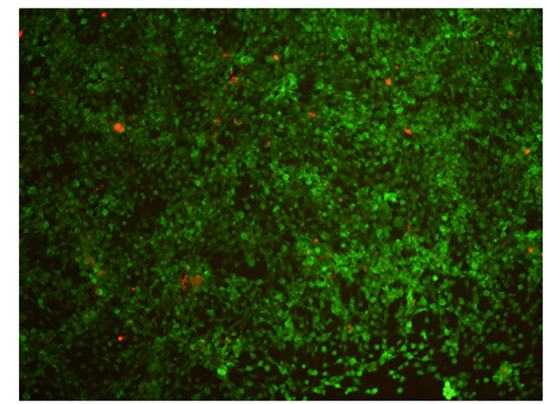

$30 \mu \mathrm{M}$

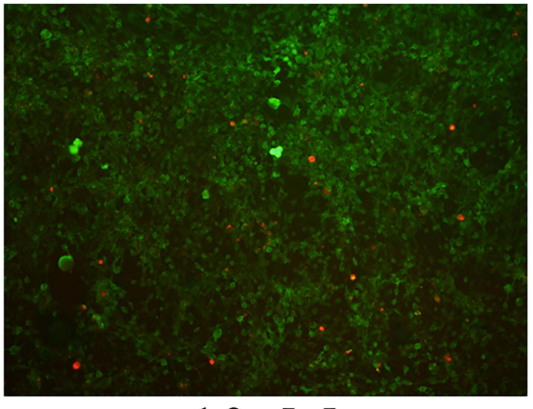

$10 \mu \mathrm{M}$

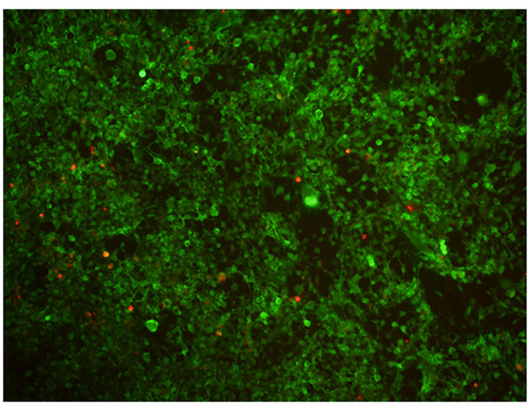

$40 \mu \mathrm{M}$

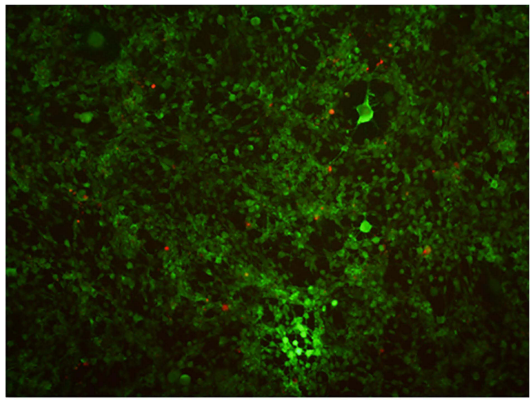

$20 \mu \mathrm{M}$

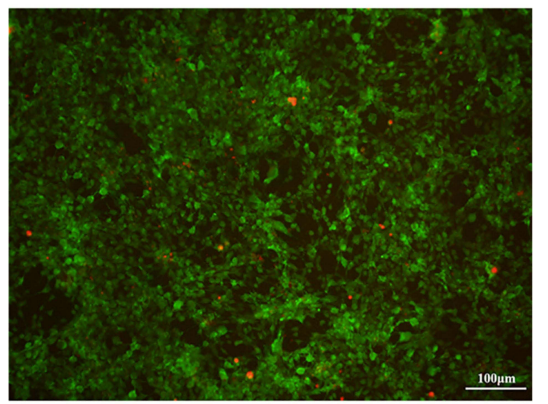

$50 \mu \mathrm{M}$

Figure I (A) CCK-8 assay was performed with different concentrations of $\mathrm{OB}(10,20,30,40,50 \mu \mathrm{M})$ for I, 3, 5,7 days and indicated that all compounds have no toxic effect on BMMs. (B) The live/dead staining under different concentrations of $\mathrm{OB}(10,20,30,40,50 \mu \mathrm{M})$ at day 7.

OB Inhibits RANKL-Mediated Activation of NF- $\kappa B$ and MAPK Signaling Pathways

Osteoclast formation requires the timely activation of MAPK and NF-KB pathways. We investigated the role of OB against these two pathways following stimulation of RANKL. In MAPK signaling pathways, administration of $O B$ significantly inhibited the increased phosphorylated level of ERK, JNK and p38 within 45 min after RANKL stimulation (Figure 5A and B). The NF-kB signaling cascades is another important downstream target required for the formaion of mature osteoclasts. Upon RANK/RANKL activation, the IkB kinase complex induces phosphorylation and degradation of $\mathrm{IkB} \alpha$, which 


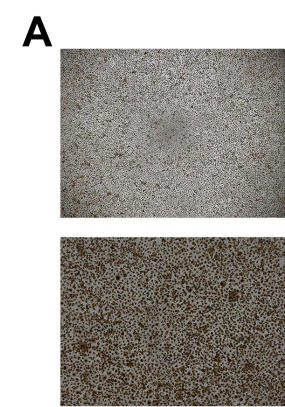

Control
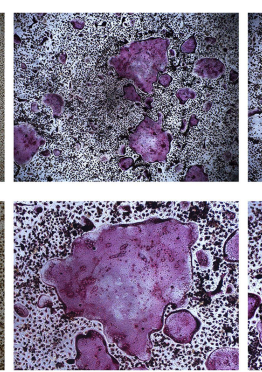

RANKL
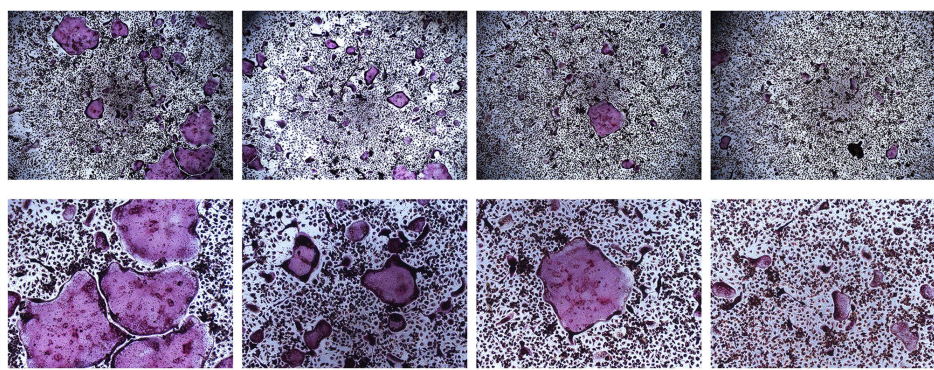

$10 \mu \mathrm{M}$

$20 \mu \mathrm{M}$
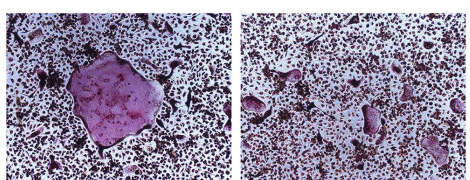

$30 \mu \mathrm{M}$

$40 \mu \mathrm{M}$
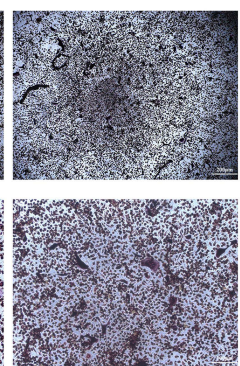

$50 \mu \mathrm{M}$

B

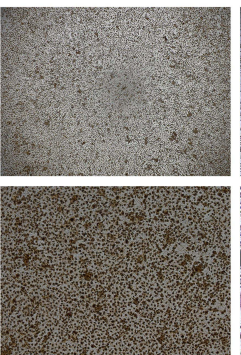

Control

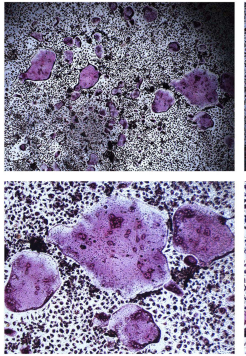

RANKL

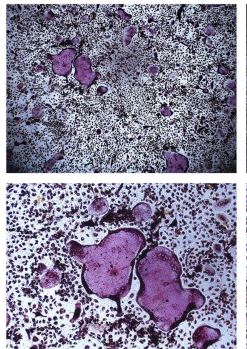

Day 1-3

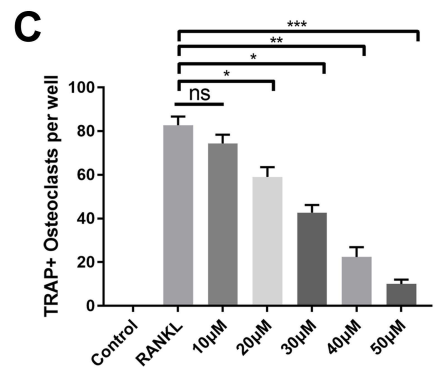

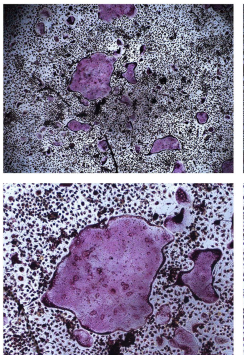

Day 3-5

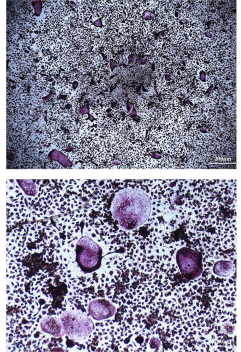

Day 1-5

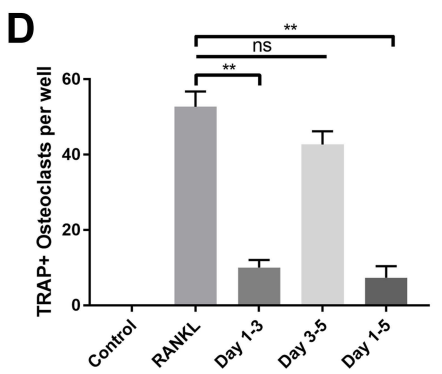

Figure 2 OB inhibits RANKL-mediated osteoclastogenesis in vitro. (A) TRAP staining showed different concentrations of OB (I0, 20, 30, 40, 50 $\mu M$ ) inhibited osteoclast formation (upper: $200 \mu \mathrm{m}$ magnification, lower: $100 \mu \mathrm{m}$ magnification). (B) TRAP staining showed the time dependent effect of OB (50 $\mu \mathrm{M})$ on osteoclast formation, on day I of $O B$ interference (sustaining until day 3 ), on day 3 of $O B$ interference (sustaining until day 5 ) and OB interference on the entire duration (upper: $200 \mu m$ magnification, lower: $100 \mu \mathrm{m}$ magnification). (C) TRAP-positive cells were quantified after administration of different concentrations of OB. (D) TRAP-positive cells were quantified after administration in different time periods. $* \mathrm{P}<0.05$; $* * \mathrm{P}<0.01$; $* * * \mathrm{P}<0.001$.

lead to the phosphorylation and accumulation of $\mathrm{p} 65$, followed by nuclear translocation and binding to the target site to stimulate related transcription factors. In our study, OB treatment inhibited degradation of $\mathrm{IkB} \alpha$ and NF- $\mathrm{\kappa B}$ p 65 phosphorylation and nuclear localization induced by RANKL (Figure 6A-C).

\section{OB Inhibits Ovariectomy (OVX)-Induced Bone Loss and Osteoclast Formation in vivo}

Based on the above results of $\mathrm{OB}$ on BMMs in vitro, we next investigated that whether $\mathrm{OB}$ also inhibits the osteoclast formation and function in vivo and play a protective effect against osteoclast-mediated bone loss caused by
OVX. Three-dimensional (3D) reconstruction showed a decreased bone mass in ovariectomized mice model and OB treatment attenuated bone loss induced by estrogen deficiency (Figure 7A). Through analyzing the bone parameters after reconstruction, we found that BV/TV, Tb. $\mathrm{N}$, and Tb.Sp were decreased in the OVX group and increased after OB administration (Figure 7B). Consistently, H\&E staining showed the trabeculae were rare and thin in the region of distal femur in the OVX group and $\mathrm{OB}$ treatment could prevent bone loss caused by OVX (Figure 8A). Additionally, TRAP staining was used to investigate the effect of $\mathrm{OB}$ on osteoclast formation in vivo. Mice treated with $O B$ showed the reduced number of osteoclasts per bone surface (N.Oc/BS) compared with OVX (Figure 8B and C). Moreover, we examined the 


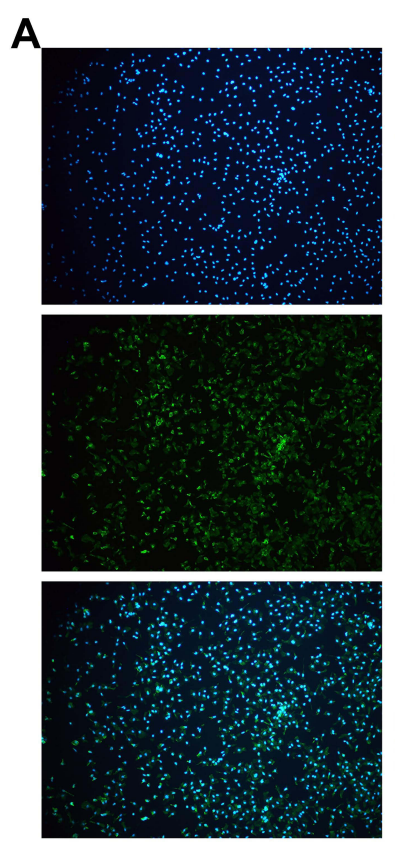

Control

B

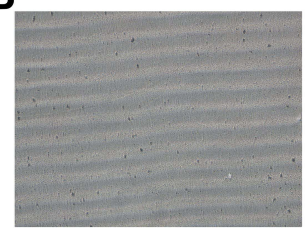

Control
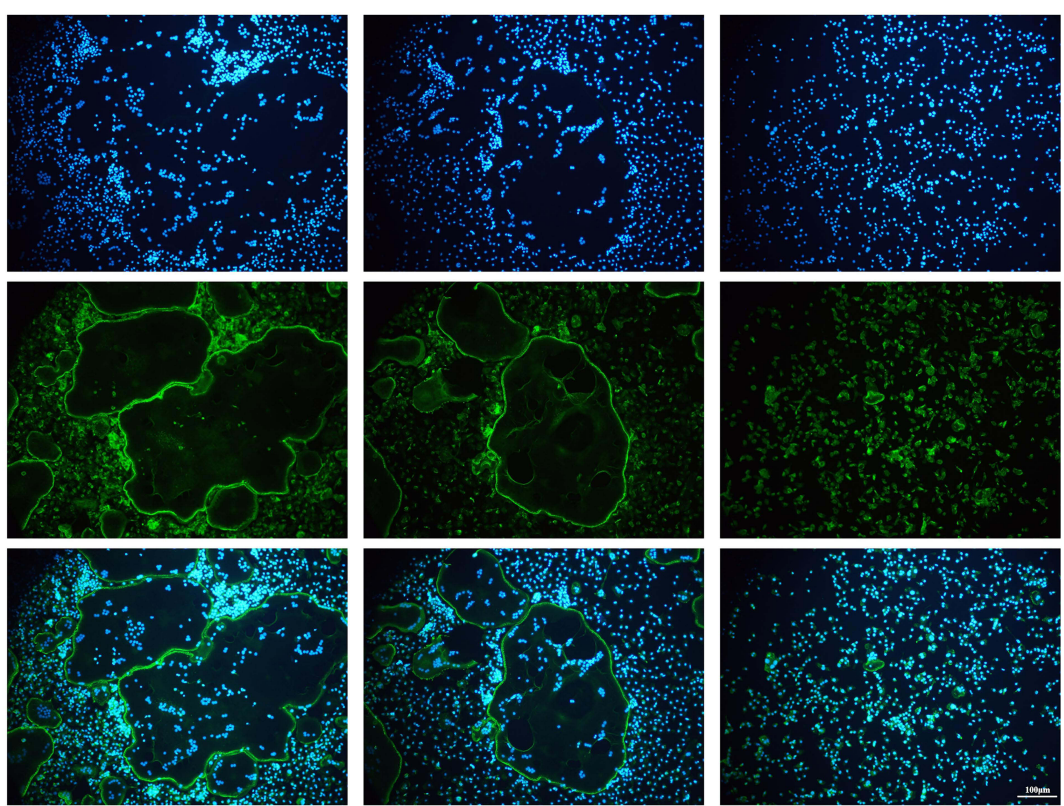

RANKL
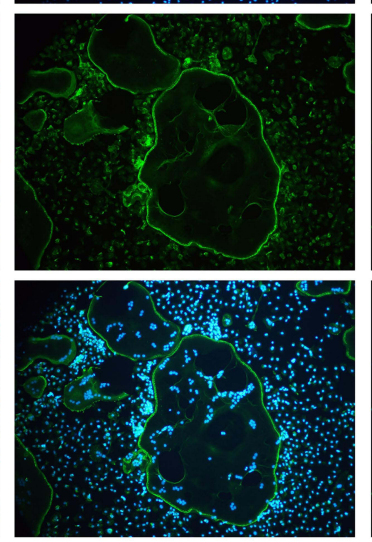

$20 u \mathrm{M}$
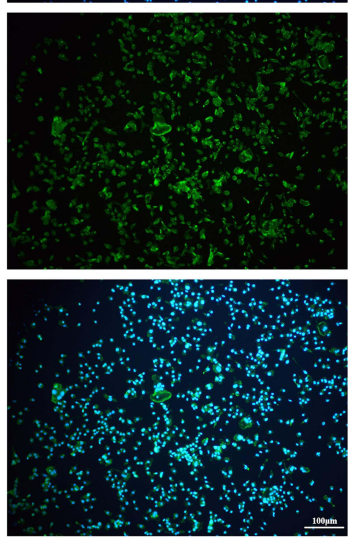

$50 \mathrm{uM}$
DAPI

\section{F-actin}

\section{Merge}

Figure 3 OB exhibits anti-resorption effect in vitro. (A) The formed podosomal actin belts as assessed by Actin-Tracker staining. (B) Representative images of pit area by osteoclasts on Osseo Assay plate. (C) Quantification of pits formation area. **P $<0.0$ I.

serum level of CTX-I, RANKL and OPG. The data of serologic markers showed that the level of CTX-I and RANKL were increased in OVX mice, whereas administration of OB decreased the CTX-I and RANKL level. Serum OPG level in OVX mice was reduced, but OB treatment did not significantly alter serum OPG levels. As a result, the RANKL/OPG ratio was reduced in OVX mice treated with $\mathrm{OB}$, as compared with OVX mice (Figure 8D).

\section{Discussion}

Bone remolding is a dynamic and constant process, which occurs continuously. During bone remolding, the bone is reabsorbed by osteoclasts and new bone formation is performed by bone-forming cells. Bone formation and bone resorption are highly coordinated under physiological conditions. In some pathological conditions, such as osteoporosis, long-term excessive formation and function of osteoclast are common. Thus, the negative regulation of osteoclasts directly affects skeletal integrity and may be a feasible strategy for preventing bone loss. ${ }^{24}$

Oroxylum indicum (L.) Vent has been used as an analgesic, antitussive and as an anti-inflammatory agent for treating coughs, bronchitis, and other diseases. ${ }^{25}$ Through modern pharmacological analysis and verification, it was found that it possesses anti-inflammatory, anticancer, antioxidant, and immunostimulant activities, indicating its potential in clinical use. ${ }^{26-29} \mathrm{OB}$ as a major bioactive constituent, has been proven to possess anticancer properties. ${ }^{18-20}$ However whether OB affects osteoclasts remains unknown. In this study, we demonstrated that $\mathrm{OB}$ could effectually inhibit osteoclast formation and function both in vitro and in vivo, and effectively attenuate bone loss induced by OVX. To our knowledge, this is the first time such an effect has been proven.

As reported in previous studies, the activation of MAPK and NF- $\mathrm{KB}$ signaling pathway has been demonstrated as important regulatory pathway in osteoclastogenesis. ${ }^{30,31}$ 
A
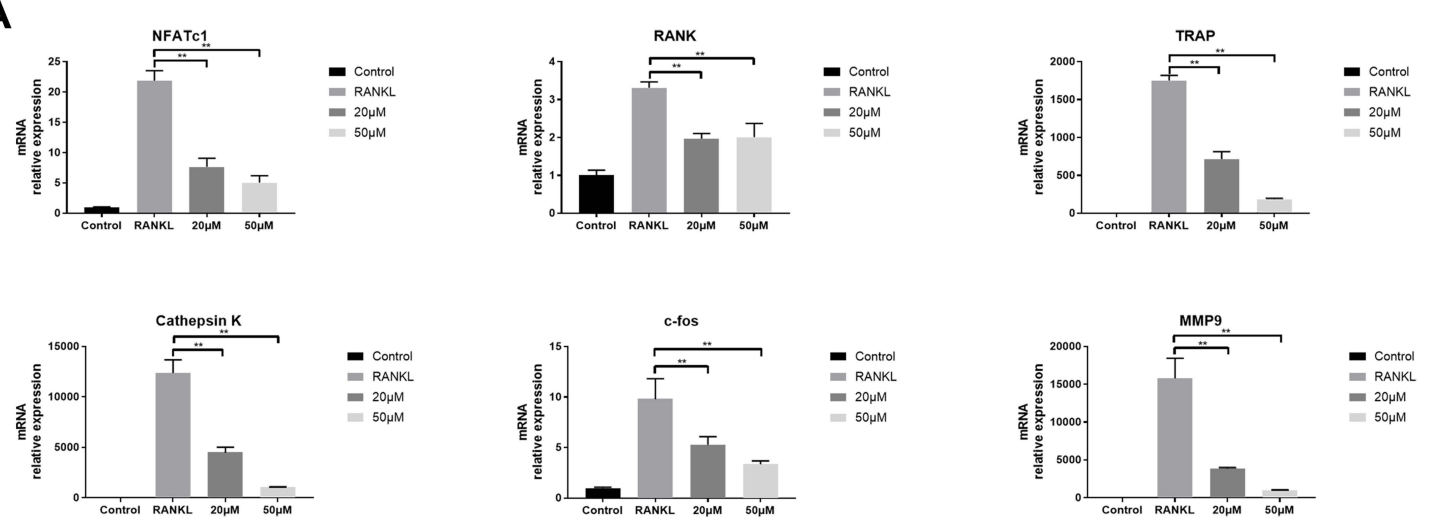

B

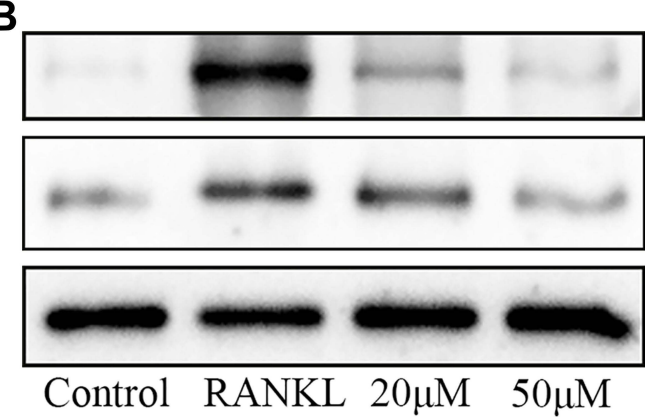

D

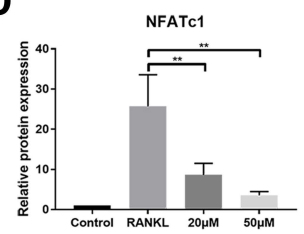

F
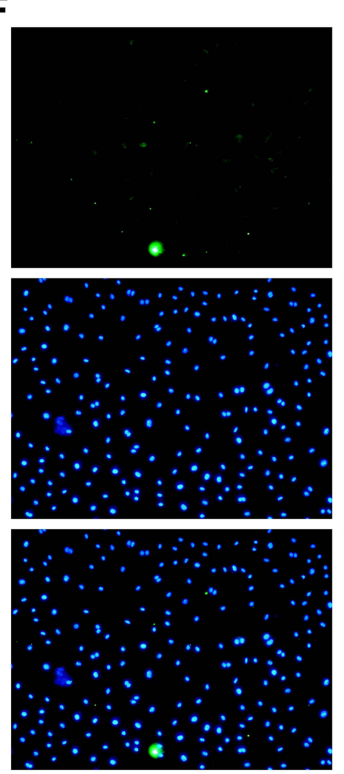

Control
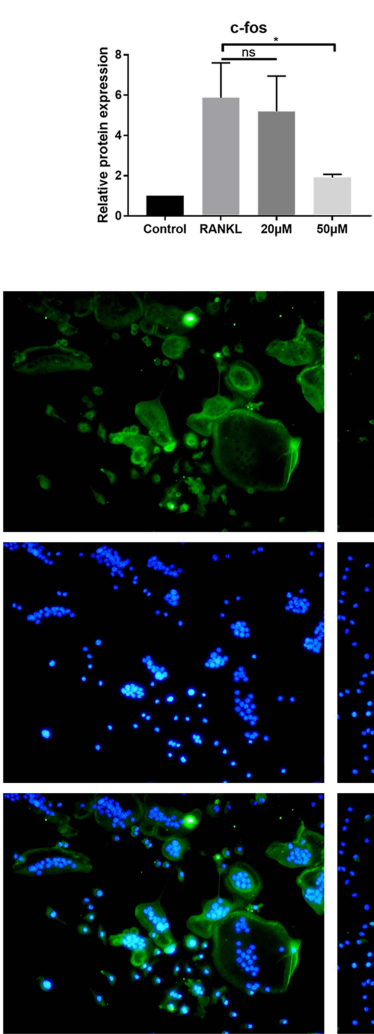

RANKL
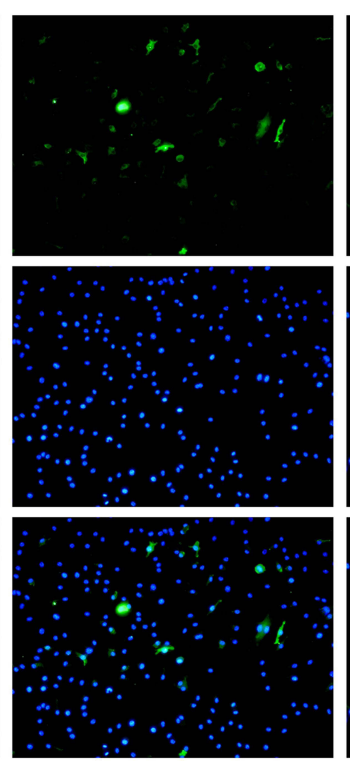

$20 \mu \mathrm{M}$

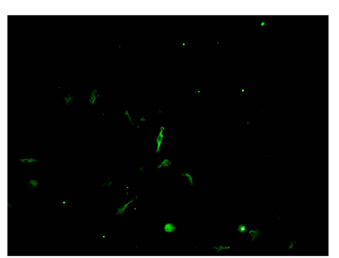

NFATc1

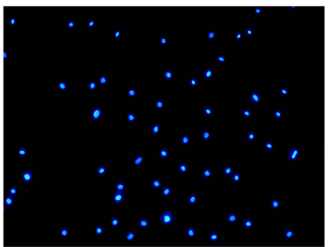

DAPI

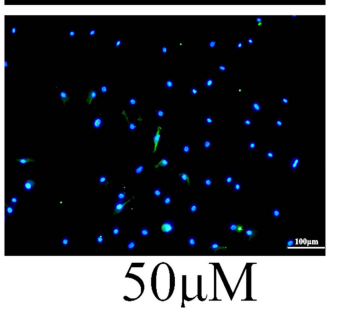

Merge
MMP9

Cathepsine K

$\beta$-actin

E
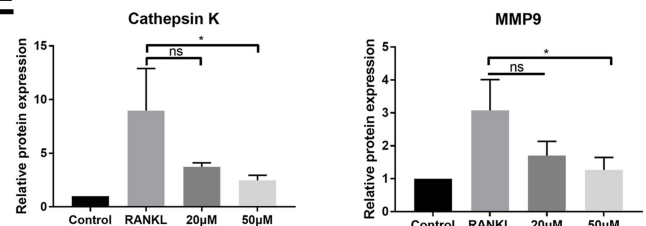

Figure $4 \mathrm{OB}$ abrogates RANKL-associated NFATcland c-fos transcription and downregulates osteoclast-related genes. (A) The RT-qPCR assay detected the relative mRNA expression of osteoclastogenesis-associated marker genes, including NFATcl, c-fos, TRAP, Rank, cathepsin K and MMP9. (B) Western-blot analysis for OB's effects on protein levels of NFATcl and c-fos. (C) Western-blot analysis for OB's effects on protein levels of MMP9 and cathepsin K. (D) Quantitative analysis of NFATcl and c-fos. (E) Quantitative analysis of MMP9 and cathepsin K. (F) Immunofluorescence images for OB's effects on protein expression of NFATcl. ns, no statistical significance; *P < 0.05 ; **P < 0.0 I. 
A

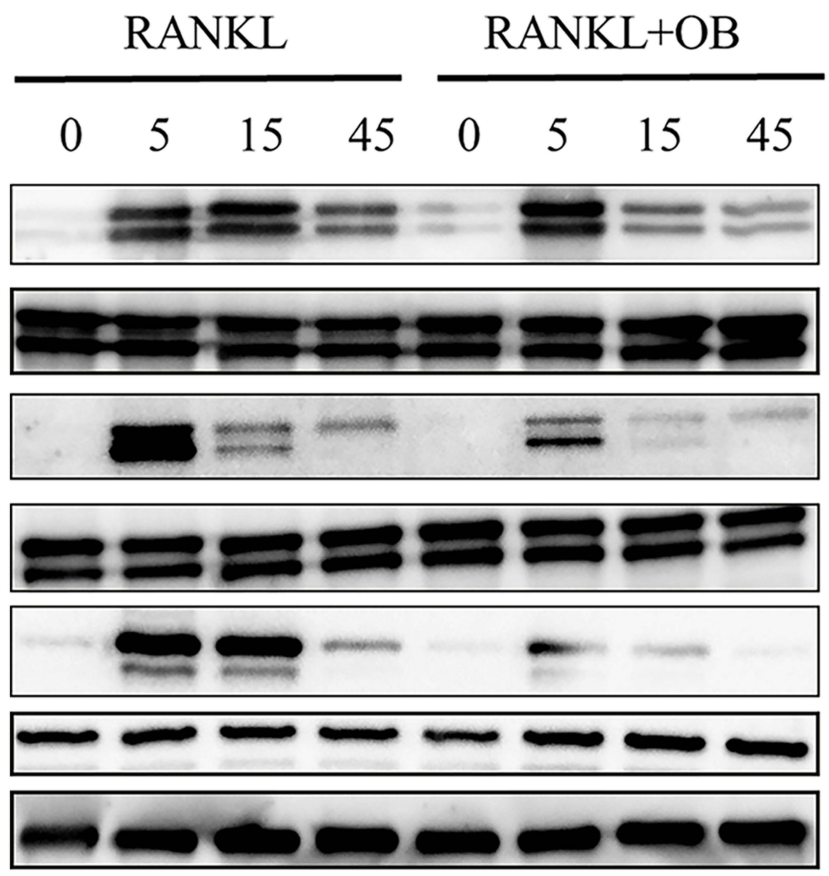

B

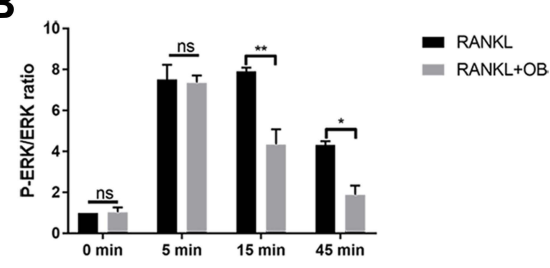

\section{ERK}

P-JNK

JNK

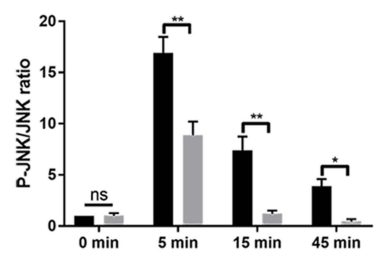

- RANKL

- RANKL+OB

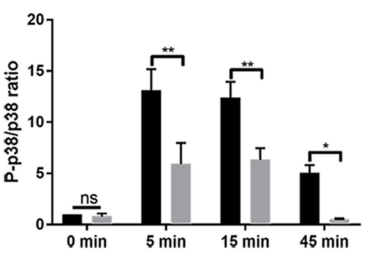

- RANKL

- RANKL+OB

$\beta$-actin

Figure $5 \mathrm{OB}$ attenuates RANKL-mediated activation of MAPK signaling pathway. BMMs were pretreated with OB (50 $\mu$ M) in FBS-free medium for I2 hours. Then, all cells were stimulated with RANKL $(50 \mathrm{ng} / \mathrm{mL})$ for $5 \mathrm{~min}, 15 \mathrm{~min}$ and $45 \mathrm{~min}$, respectively. (A) Western-blot analysis detected effect of OB on the phosphorylated level of ERK, JNK and p38 after RANKL induction. (B) Quantitative analysis revealed the phosphorylated ERK, JNK and p38 to total ERK, JNK and p38. ns, no statistical significance; *P < 0.05 ; $* * \mathrm{P}<0.01$.

Extracellular signal-regulated kinase (ERK), c-Jun N-terminal kinase (JNK), and MAPK p38 constitute the MAPK signaling pathway. During RANKL-induced osteoclastogenesis, the phosphorylation of ERK and JNK initial transcription of c-fos, contributes to osteoclast lineage commitment. $^{32,33}$ The activated p38 directly stimulates transcription of NFATc1, which is considered as a key event in the determination of the fate of osteoclasts. ${ }^{7}$ In our study, we found that pretreatment with $\mathrm{OB}$ could significantly inhibit the increase in phosphorylation levels of all three MAPKs induced by RANKL in different degrees. Phosphorylation of $\mathrm{I} \kappa \mathrm{B} \alpha$ was increased after RANKL stimulation, which in turn leads to the phosphorylation and accumulation of P65, followed by nuclear translocation and binding to a DNA target site to stimulate associated transcription factors. ${ }^{31}$ According to our data, the phosphorylation level of I $\kappa \mathrm{B}$ and P65 was suppressed by $\mathrm{OB}$ and the accumulation of $\mathrm{P} 65$ in nuclear was reduced. These data suggested $\mathrm{OB}$ inhibits the increase in phosphorylation levels of both MAPK and NF-kB signaling pathways, resulting in the inhibition of osteoclast formation and function.

As mention above, c-fos and NFATc1, the downstream targets of MAPK and NF- $\mathrm{B}$ signaling pathway, are two indispensable regulatory factors in osteoclast formation.
c-Fos originates from the FOS family proteins, which belongs to the activator protein 1 (AP-1) transcription factor group. During osteoclast differentiation, c-Fos is induced at an early differentiation stage, which binds to NFATc1 promoter to promote NFATc1 expression and acts as a driver to trigger the shift to osteoclast or macrophage differentiation from a common progenitor. c-Fos mutant mice showed osteopetrotic phenotype with reduced number of TRAPpositive osteoclasts. ${ }^{34}$ NFATc1, another key transcription factor regulating several osteoclast-specific genes such as TRAP, cathepsin K and MMP9, through the cooperation with c-Fos, which are the key matrix-degrading enzymes for bone resorption. $^{35-37}$ The indispensable effect of NFATc1 in osteoclastogenesis has been well demonstrated in some studies. The upregulated expression of NFATc1 could significantly stimulate osteoclast differentiation even though in absence of RANKL and osteoclast-specific conditional NFATc1-deficient mice resulted in osteopetrosis owing to the defective of osteoclast differentiation. ${ }^{38,39}$ In our current study, the upregulated expression of c-fos, cathepsin $\mathrm{K}$, MMP9 and NFATc1 stimulated by RANKL were reduced by the administration of OB.

In vivo, as a serum biomarker of bone resorption, the serum CTX-I level was increased in OVX mice and 
A

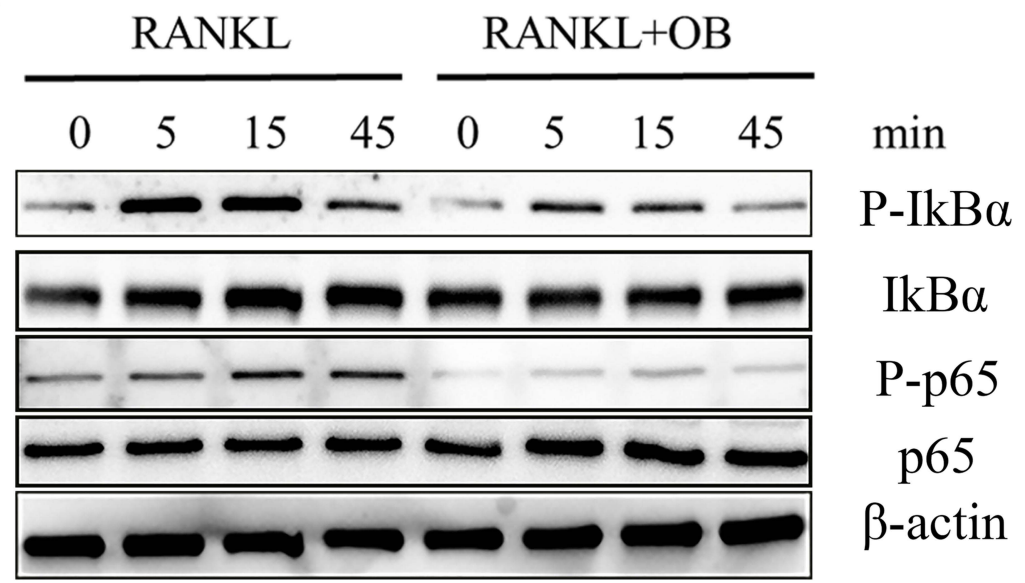

B

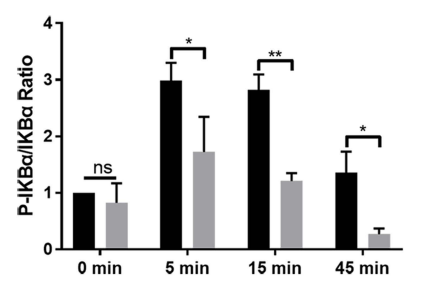

- RANKL
Rankl+OB

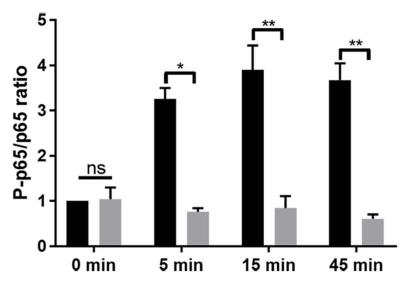

- RANKL

- Rankl+OB

C
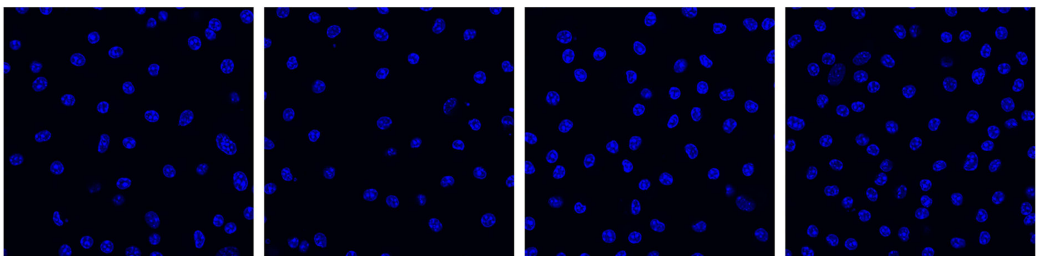

DAPI
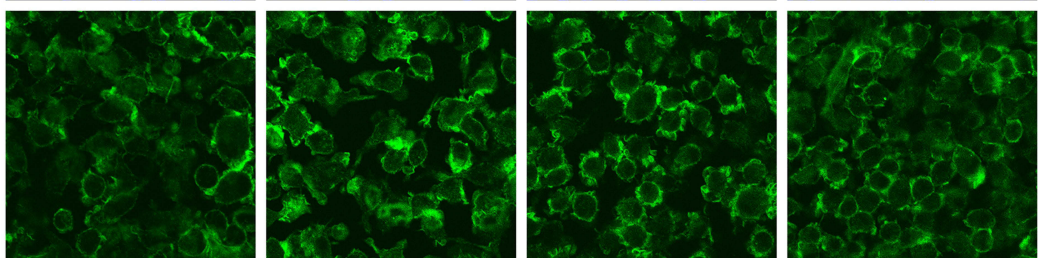

Actin
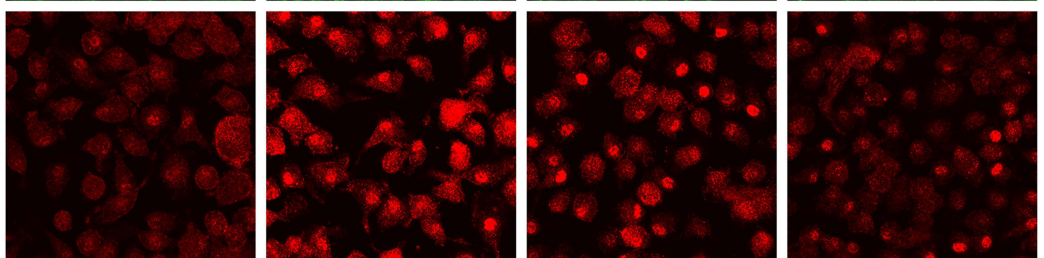

P-p65
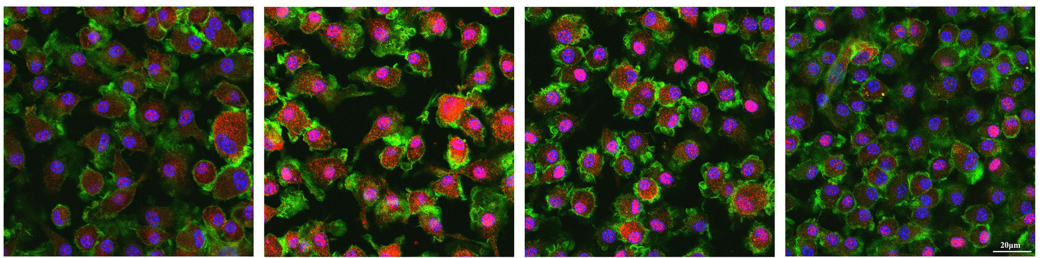

Merge

Control

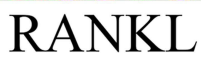

$20 \mu \mathrm{M}$

$50 \mu \mathrm{M}$

Figure $6 \mathrm{OB}$ attenuates RANKL-mediated activation of NF- $\mathrm{KB}$ signaling pathway. BMMs were pretreated with $\mathrm{OB}(50 \mu \mathrm{M})$ in FBS-free medium for 12 hours. Then, all cells were stimulated with RANKL $(50 \mathrm{ng} / \mathrm{mL})$ for $5 \mathrm{~min}, 15 \mathrm{~min}$ and $45 \mathrm{~min}$, respectively. (A) Western-blot analysis detected effect of OB on the phosphorylated level of $\mathrm{lkB}-\alpha$ and p65 associated with RANKL stimulation. (B) Quantitative analysis of phosphorylated lkB- $\alpha$ and $p 65$ to total lkB- $\alpha$ and $p 65$. (C) Confocal images detected nuclear of $p 65$ following RANKL stimulation without or with $\mathrm{OB}$ treatment. ns, no statistical significance; $* \mathrm{P}<0.05$; $* * \mathrm{P}<0.01$. 
A
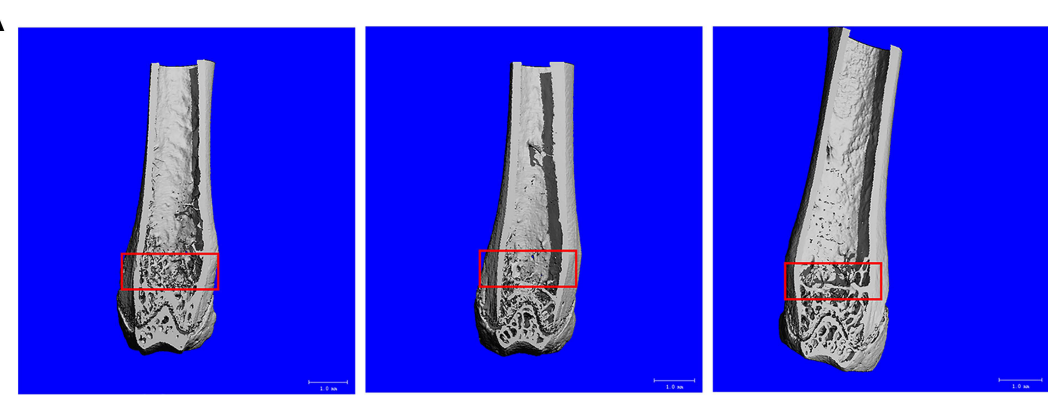

coronal view
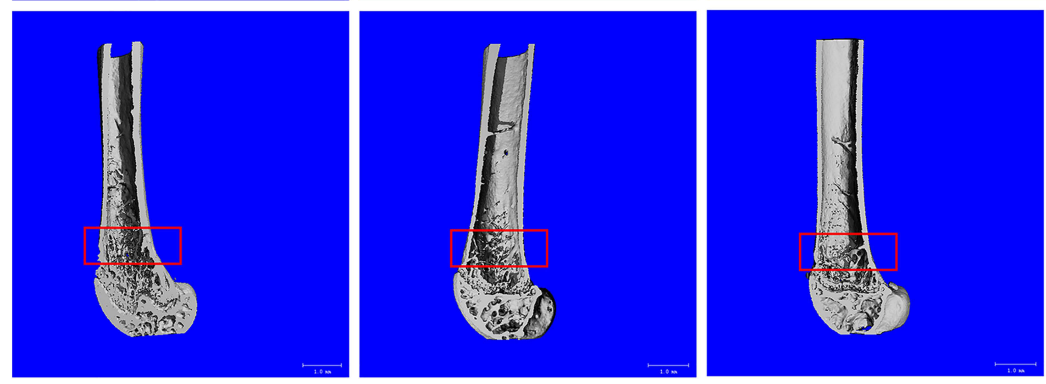

sagittal view

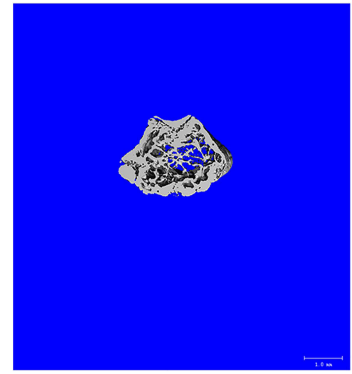

Sham

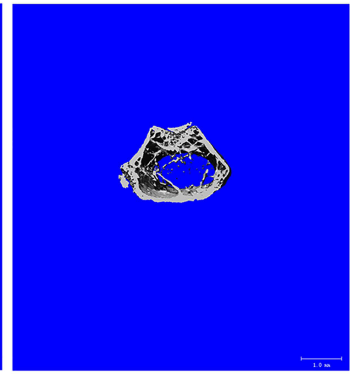

OVX

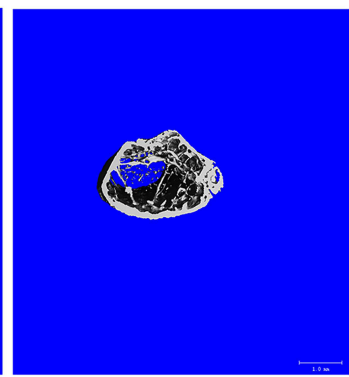

OB

B
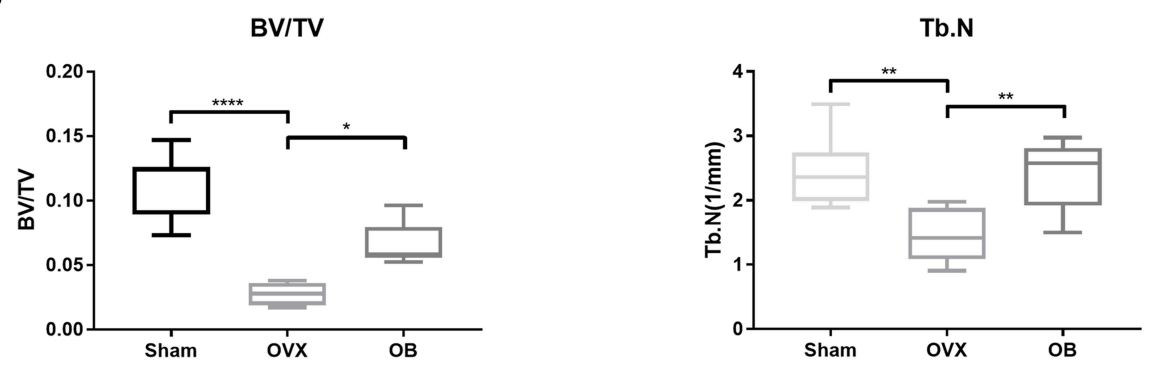

Tb.Th
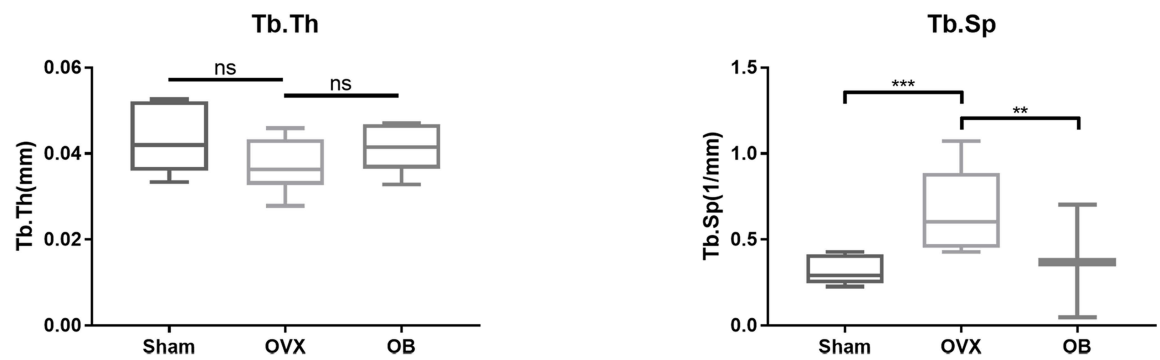

Figure $7 \mathrm{OB}$ inhibits ovariectomy (OVX)-mediated bone loss in vivo. (A) Representative 3D reconstruction micro-CT images of the trabecular bone of distal femoral metaphysis, showing the protective effect of $\mathrm{OB}$ against bone loss. (B) Morphometric parameters of trabecular structure: trabecular bone volume/tissue volume (BV/TV), trabecular number (Tb.N), trabecular thickness (Tb.Th), and trabecular separation (Tb.Sp). ns, no statistical significance; *P $<0.05 ; * * \mathrm{P}<0.01 ; * * * \mathrm{P}<0.001 ; * * * * \mathrm{P}<0.0001$. 


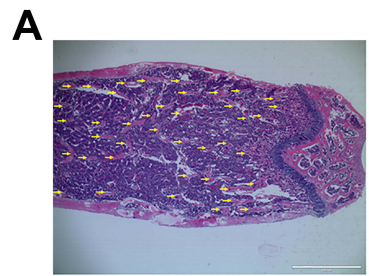

Sham

B
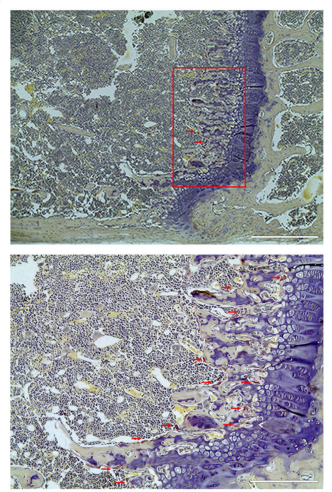

Sham

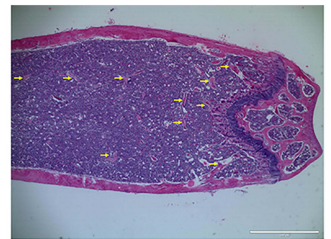

OVX

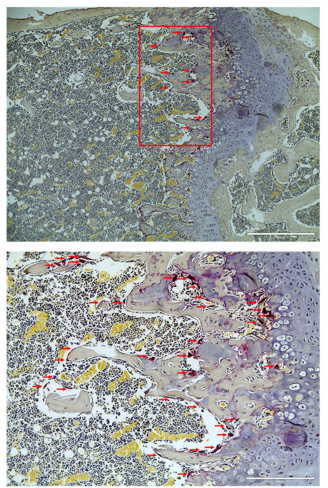

OVX

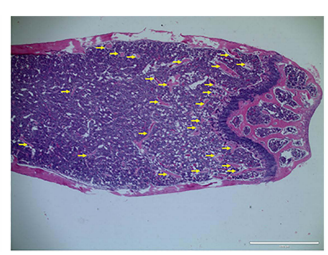

$\mathrm{OB}$

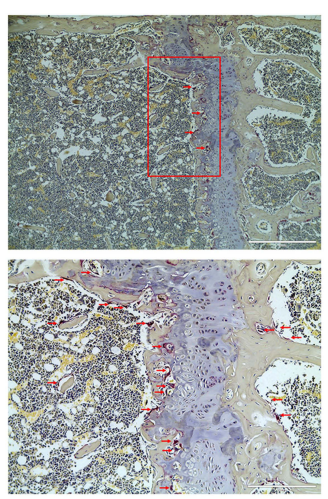

OB
C

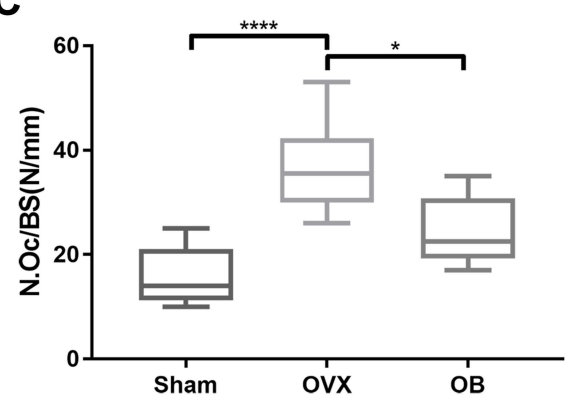

D
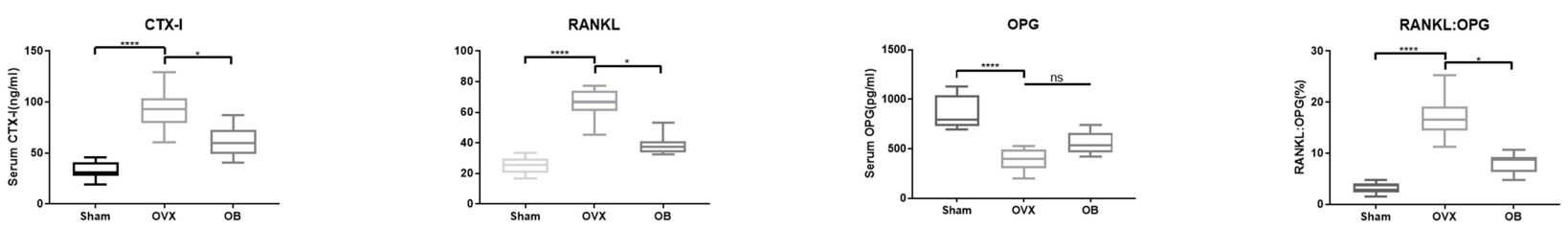

Figure 8 OB Suppresses osteoclast formation and bone resorption in OVX mice. (A) Representative photographs of femoral samples after HE-stained sections (changes in trabeculae represented by yellow arrows). (B) TRAP-stained sections of femoral samples. Second row of images are the magnified areas of the highlighted sections (changes in osteoclasts represented by red arrows). (C) Quantitative histomorphometric analysis of TRAP-positive osteoclasts. (D) The serum level of CTX-I, OPG, RANKL and the RANKL: OPG ratio. ns, no statistical significance; $* \mathrm{P}<0.05$; $* * * * \mathrm{P}<0.0001$.

decreased after administration of $\mathrm{OB}$, which was consistent with the results of our in vitro study. As mentioned above, RANKL is the primary signal for osteoclastic differentiation, while OPG acts as a decoy receptor that competitively binds to RANKL and thus interrupts the RANKL-RANK activation and then impairs the differentiation and maturation of osteoclasts. ${ }^{40}$ The serologic results showed that the upregulated RANKL in OVX mice were regulated by $\mathrm{OB}$ administration, leading to alteration of RANKL/OPG ratio, which indicated that OB regulated the differentiation and maturation of osteoclasts through RANKL/OPG system in vivo.

We do acknowledge the limitations in our study. Firstly, our study clarified that OB showed excellent efficacy on inhibiting osteoclast differentiation. However, the influence of $\mathrm{OB}$ on osteoblasts still needs to be verified. The molecular targets of OB regarding osteoclast differentiation also need to be clarified in future studies.
To our knowledge, our study is the first to demonstrate that $\mathrm{OB}$ possesses protective effects against bone loss resulting from ovariectomy, by suppressing the expression of c-fos and NFATc1, through regulating the activation of NF- $\mathrm{BB}$ and MAPK in RANKLinduced osteoclast differentiation. Taken together, these findings indicate that $\mathrm{OB}$ may serve as a latent therapeutic strategy for osteoporosis. The antiosteoclastogenic activity of $\mathrm{OB}$ under clinical settings should be addressed in future.

\section{Acknowledgments}

This work was supported by the National Natural Science Foundation of China (81871742).

\section{Author Contributions}

All of the authors contributed to data analysis, drafting and/or revising the article, and have given the final 
approvals for this manuscript, agreed to the submitted journal, and to be accountable for all aspects of the work.

\section{Disclosure}

The authors declare that they have no competing interests in this work.

\section{References}

1. Svedbom A, Hernlund $\mathrm{E}$, Ivergård $\mathrm{M}$, et al. Osteoporosis in the European Union: a compendium of country-specific reports. Arch Osteoporos. 2013;8(1-2):137. doi:10.1007/s11657-013-0137-0

2. Lane NE. Epidemiology, etiology, and diagnosis of osteoporosis. $A m$ $J$ Obstet Gynecol. 2006;194(2 Suppl):S3-11. doi:10.1016/j. ajog.2005.08.047

3. Wang T, Liu Q, Zhou L, et al. Andrographolide inhibits ovariectomy-induced bone loss via the suppression of RANKL signaling pathways. Int $J$ Mol Sci. 2015;16(11):27470-27481. doi:10.3390/ijms 161126039

4. Xu H, Liu T, Jia Y, et al. (-)-Epigallocatechin-3-gallate inhibits osteoclastogenesis by blocking RANKL-RANK interaction and suppressing $\mathrm{NF}-\kappa \mathrm{B}$ and MAPK signaling pathways. Int Immunopharmacol. 2021;95:107464. doi:10.1016/j.intimp.2021.107464

5. Mun SH, Park PSU, Park-Min KH. The M-CSF receptor in osteoclasts and beyond. Exp Mol Med. 2020;52(8):1239-1254. doi:10.1038/s12276-020-0484-z

6. Lee SE, Chung WJ, Kwak HB, et al. Tumor necrosis factor-alpha supports the survival of osteoclasts through the activation of Akt and ERK. J Biol Chem. 2001;276(52):49343-49349. doi:10.1074/jbc. M103642200

7. Takayanagi H, Kim S, Koga T, et al. Induction and activation of the transcription factor NFATc1 (NFAT2) integrate RANKL signaling in terminal differentiation of osteoclasts. Dev Cell. 2002;3(6):889-901. doi:10.1016/s1534-5807(02)00369-6

8. Jiang L, Numonov S, Bobakulov K, Qureshi MN, Zhao H, Aisa HA. Phytochemical profiling and evaluation of pharmacological activities of Hypericum scabrum L. Molecules. 2015;20(6):11257-11271. doi:10.3390/molecules200611257

9. Mori T, Kobayashi H, Ono R, Tajima T. [Therapeutic result and roentgenological prognosis of cervical spondylosis after Cloward surgery]. Seikei Geka. 1970;21(8):618-625. Japanese.

10. Nijveldt RJ, van Nood E, van Hoorn DE, Boelens PG, van Norren K, van Leeuwen PA. Flavonoids: a review of probable mechanisms of action and potential applications. Am J Clin Nutr. 2001;74 (4):418-425. doi:10.1093/ajen/74.4.418

11. Zhang X, Liu T, Huang Y, Wismeijer D, Liu Y. Icariin: does it have an osteoinductive potential for bone tissue engineering? Phytother Res. 2014;28(4):498-509. doi:10.1002/ptr.5027

12. Wang Z, Wang D, Yang D, Zhen W, Zhang J, Peng S. The effect of icariin on bone metabolism and its potential clinical application. Osteoporosis Int. 2018;29(3):535-544. doi:10.1007/s00198-017-4255-1

13. Huang JM, Bao Y, Xiang W, et al. Icariin regulates the bidirectional differentiation of bone marrow mesenchymal stem cells through canonical Wnt signaling pathway. eCAM. 2017;2017:8085325. doi:10.1155/2017/8085325

14. Zhu W, Yin Z, Zhang Q, et al. Proanthocyanidins inhibit osteoclast formation and function by inhibiting the NF- $\mathrm{BB}$ and JNK signaling pathways during osteoporosis treatment. Biochem Biophys Res Commun. 2019;509(1):294-300. doi:10.1016/j.bbrc.2018.12.125

15. Lee J, Noh AL, Zheng T, Kang JH, Yim M. Eriodicyol inhibits osteoclast differentiation and ovariectomy-induced bone loss in vivo. Exp Cell Res. 2015;339(2):380-388. doi:10.1016/j.yexcr.2015.10.001
16. Lu L, Rao L, Jia H, et al. Baicalin positively regulates osteoclast function by activating MAPK/Mitf signalling. J Cell Mol Med. 2017;21(7):1361-1372. doi:10.1111/jcmm.13066

17. Qiu J, Wang D, Zhang Y, Dong J, Wang J, Niu X. Molecular modeling reveals the novel inhibition mechanism and binding mode of three natural compounds to staphylococcal $\alpha$-hemolysin. PLoS One. 2013;8(11):e80197. doi:10.1371/journal.pone.0080197

18. Yang $\mathrm{P}, \mathrm{Fu} \mathrm{S}$, Cao Z, et al. Oroxin B selectively induces tumor-suppressive ER stress and concurrently inhibits tumor-adaptive ER stress in B-lymphoma cells for effective anti-lymphoma therapy. Toxicol Appl Pharmacol. 2015;288 (2):269-279. doi:10.1016/j.taap.2015.07.026

19. Li NN, Meng XS, Bao YR, Wang S, Li TJ. Evidence for the Involvement of COX-2/VEGF and PTEN/Pl3K/AKT pathway the mechanism of oroxin $\mathrm{b}$ treated liver cancer. Pharmacogn Mag. 2018;14(54):207-213. doi:10.4103/pm.pm_119_17

20. Li N, Men W, Zheng Y, Wang H, Meng X. Oroxin B induces apoptosis by down-regulating MicroRNA-221 resulting in the inactivation of the PTEN/PI3K/AKT pathway in liver cancer. Molecules. 2019;24(23):4384. doi:10.3390/molecules24234384

21. Guan H, Zhao L, Cao H, Chen A, Xiao J. Epoxyeicosanoids suppress osteoclastogenesis and prevent ovariectomy-induced bone loss. FASEB $j$. 2015;29(3):1092-1101. doi:10.1096/fj.14-262055

22. Sakai A, Nishida S, Nishida S, et al. 1alpha-Hydroxyvitamin D3 suppresses trabecular bone resorption by inhibiting osteoclastogenic potential in bone marrow cells after ovariectomy in mice. $J$ Bone Miner Metab. 2001;19(5):277-286. doi:10.1007/s007740170011

23. Zhan Y, Liang J, Tian K, et al. Vindoline Inhibits RANKL-induced osteoclastogenesis and prevents ovariectomy-induced bone loss in mice. Front Pharmacol. 2019;10:1587. doi:10.3389/fphar.2019.01587

24. Jeong E, Choi HK, Park JH, Lee SY. STAC2 negatively regulates osteoclast formation by targeting the RANK signaling complex. Cell Death Differ. 2018;25(8):1364-1374. doi:10.1038/s41418-017-0048-5

25. Lalrinzuali K, Vabeiryureilai M, Jagetia GC. Topical application of stem bark ethanol extract of Sonapatha, Oroxylum indicum (L.) Kurz accelerates healing of deep dermal excision wound in Swiss albino mice. J Ethnopharmacol. 2018;227:290-299. doi:10.1016/j.jep.2018.08.018

26. Doshi K, Ilanchezhian R, Acharya R, Patel BR, Ravishankar B. Antiinflammatory activity of root bark and stem bark of Shyonaka. J Ayurveda Integr Med. 2012;3(4):194-197. doi:10.4103/09759476.104434

27. Siriwatanametanon N, Fiebich BL, Efferth T, Prieto JM, Heinrich M. Traditionally used Thai medicinal plants: in vitro anti-inflammatory, anticancer and antioxidant activities. J Ethnopharmacol. 2010;130 (2):196-207. doi:10.1016/j.jep.2010.04.036

28. Zaveri M, Gohil P, Jain S. Immunostimulant activity of n-butanol fraction of root bark of Oroxylum indicum, vent. J Immunotoxicol. 2006;3(2):83-99. doi:10.1080/15476910600725942

29. Nik Salleh NNH, Othman FA, Kamarudin NA, Tan SC. The biological activities and therapeutic potentials of baicalein extracted from oroxylum indicum: a systematic review. Molecules. 2020;25 (23):5677. doi:10.3390/molecules 25235677

30. Pearson G, Robinson F, Beers Gibson T, et al. Mitogen-activated protein (MAP) kinase pathways: regulation and physiological functions. Endocr Rev. 2001;22(2):153-183. doi:10.1210/ edrv.22.2.0428

31. Abu-Amer Y. NF-kB signaling and bone resorption. Osteoporosis Int. 2013;24(9):2377-2386. doi:10.1007/s00198-013-2313-x

32. Lee MS, Kim HS, Yeon JT, et al. GM-CSF regulates fusion of mononuclear osteoclasts into bone-resorbing osteoclasts by activating the Ras/ERK pathway. J Immunol. 2009;183(5):3390-3399. doi:10.4049/jimmunol.0804314

33. Chang EJ, Ha J, Huang H, et al. The JNK-dependent CaMK pathway restrains the reversion of committed cells during osteoclast differentiation. J Cell Sci. 2008;121(Pt 15):2555-2564. doi:10.1242/ jcs. 028217 
34. Krum SA. Direct transcriptional targets of sex steroid hormones in bone. J Cell Biochem. 2011;112(2):401-408. doi:10.1002/jcb.22970

35. Kim K, Kim JH, Lee J, et al. Nuclear factor of activated T cells c1 induces osteoclast-associated receptor gene expression during tumor necrosis factor-related activation-induced cytokine-mediated osteoclastogenesis. $J$ Biol Chem. 2005;280(42):35209-35216. doi:10.1074/jbc.M505815200

36. Kim Y, Sato K, Asagiri M, Morita I, Soma K, Takayanagi H. Contribution of nuclear factor of activated $\mathrm{T}$ cells $\mathrm{cl}$ to the transcriptional control of immunoreceptor osteoclast-associated receptor but not triggering receptor expressed by myeloid cells-2 during osteoclastogenesis. J Biol Chem. 2005;280(38):32905-32913. doi:10.1074/jbc.M505820200
37. Edwards JR, Mundy GR. Advances in osteoclast biology: old findings and new insights from mouse models. Nat Rev Rheumatol. 2011;7(4):235-243. doi:10.1038/nrrheum.2011.23

38. Winslow MM, Pan M, Starbuck M, et al. Calcineurin/NFAT signaling in osteoblasts regulates bone mass. Dev Cell. 2006;10(6):771-782. doi:10.1016/j.devcel.2006.04.006

39. Kim JH, Kim N. Regulation of NFATc1 in osteoclast differentiation. J Bone Metab. 2014;21(4):233-241. doi:10.11005/jbm.2014.21.4.233

40. El Kholy K, Freire M, Chen T, Van Dyke TE. Resolvin E1 promotes bone preservation under inflammatory conditions. Front Immunol. 2018;9:1300. doi:10.3389/fimmu.2018.01300

\section{Publish your work in this journal}

Drug Design, Development and Therapy is an international, peerreviewed open-access journal that spans the spectrum of drug design and development through to clinical applications. Clinical outcomes, patient safety, and programs for the development and effective, safe, and sustained use of medicines are a feature of the journal, which has also been accepted for indexing on PubMed Central. The manuscript management system is completely online and includes a very quick and fair peer-review system, which is all easy to use. Visit http://www. dovepress.com/testimonials.php to read real quotes from published authors. 